\title{
Olfactory Neuroblastoma of the Sinonasal Tract with Prominent Orbital Protrusion: A Case Report and Literature Review
}

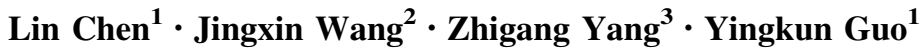

Received: 28 October 2020/Accepted: 28 December 2020/Published online: 27 January 2021

(C) The Author(s) 2021

\begin{abstract}
Olfactory neuroblastoma (ONB) is a rare malignant neuroectodermal tumor of the nasal cavity. Olfactory neuroblastoma centered in the posterior right orbit with prominent orbital protrusion is even rare. Grading ONB is extremely important as individualized treatment plans must be formulated according to tumor grade. We report the case of a 67-year-old female who presented with the chief complaints of persistent nasal congestion with intermittent epistaxis and unilateral proptosis over the past five years. Radiological imaging was suggestive of a large heterogeneous mass in the right superior nasal cavity with extensions into the right medial orbit, nasopharynx, the right maxillary sinus, the anterior cranial fossa, right ethmoidal, frontal and bilateral sphenoidal sinuses, as well as into the right frontal lobe. Assessment of the radiologic features revealed the diagnostic possibility of olfactory neuroblastoma. A
\end{abstract}

Lin Chen and Jingxin Wang have contributed equally to this work.

Zhigang Yang

yangzg666@163.com

$\triangle$ Yingkun Guo

gykpanda@163.com

1 Department of Radiology, West China Second University Hospital, Sichuan University, Key Laboratory of Obstetric and Gynecologic and Pediatric Diseases and Birth Defects of Ministry of Education, 20\# Renmin Road, Chengdu 610041, Sichuan, China

2 Department of Ultrasound, West China Second University Hospital, Sichuan University, Key Laboratory of Obstetric and Gynecologic and Pediatric Diseases and Birth Defects of Ministry of Education, 20\# Renmin Road, Chengdu 610041, Sichuan, China

3 Department of Radiology, West China Hospital, Sichuan University, 37\# Guo Xue Xiang, Chengdu 610041, Sichuan, China nasopharyngeal biopsy confirmed an olfactory neuroblastoma. Frontal osteoplastic craniotomy and excision of the intracranial part of the tumor from above and transnasal endoscopic removal of the mass in the nasal cavities, paranasal sinuses and right medial orbit from below was done. Evaluation of histopathological characteristics and immunohistochemical findings revealed a diagnosis of WHO grade IV olfactory neuroblastoma. Because of poor economic condition, the patient did not take adjuvant radiotherapy and chemoradiation and post-operative examination. We report a huge ONB centered in the posterior right orbit with prominent orbital protrusion. Magnetic resonance image and computed tomography are helpful for evaluating the appearance and the extent of ONB, as well as grading this tumor, which may aid therapeutic decisions and improve survival.

Keywords Olfactory neuroblastoma .

Magnetic resonance imaging - Computed tomography ·

Pathology

$\begin{array}{ll}\text { Abbreviations } \\ \text { ONB } & \text { Olfactory neuroblastoma } \\ \text { MRI } & \text { Magnetic resonance image } \\ \text { CT } & \text { Computed tomography } \\ \text { HIV } & \text { Human immunodeficiency virus } \\ \text { HBsAg } & \text { Hepatitis B surface antigen } \\ \text { N:C } & \text { Nuclear:cytoplasmic ratio } \\ \text { NSE } & \text { Neuron-specific enolase } \\ \text { CK } & \text { Cytokeratin } \\ \text { SMA } & \text { Smooth muscle actin } \\ \text { EMA } & \text { Epithelial membrane antigen } \\ \text { SIADH } & \text { Syndrome of inappropriate antidiuretic } \\ & \text { hormone secretion }\end{array}$




\section{ACTH Adrenocorticotropic hormone \\ CEA Carcinoembryonic antigen \\ DKI Magnetic resonance diffusion kurtosis imaging}

DCE-MRI Dynamic contrast enhanced MRI

\section{Background}

Olfactory neuroblastoma (ONB), also called as esthesioneuroblastoma, is a rare malignant neoplasm of the nasal cavity arising from the olfactory neuroepithelium. It accounts for 3-5\% of all nasal and sinonasal malignancies with no race or gender predilection [1]. ONB has a bimodal distribution and tends to occur around the second and sixth decade of life [2]. It has an incidence of 0.4 per million of population and ONB centered in the posterior right orbit with prominent orbital protrusion is even rare. Keeping in mind the rarity of ONB and the diagnostic difficulty, we present a unusual case of ONB with prominent orbital protrusion and discuss the differentiation from other nasal cavity neoplasms as emphasized in the literature review.

\section{Case presentation}

\section{History}

A 67-year-old female was presented to the Otolaryngology department with unilateral prominent orbital protrusion and a mass in the right nasal cavity, which caused persistent nasal congestion with intermittent epistaxis over 5 years. Three years earlier she had been treated for suspected chronic rhinitis without resolution of symptoms. She came for further treatment with progressive enlargement of the mass and worsening of symptoms over the last 2 years.

\section{Clinical Exam}

A nasal exam was performed using rigid endoscopy, which revealed a large mass, medial to the middle turbinate in the right nasal cavity. The mass extended to the choana, but did not appear to extend into the nasopharynx clinically. The lesion was pink and lobulated with a rubbery, non-friable texture. No lesions were identified in the left nasal cavity. Examination of visual acuity revealed no visual impairment. She had no history of headache, dizziness, or diplopia. Her blood profile for biochemistry and hematology was within normal limits. Tests for human immunodeficiency virus (HIV), hepatitis B surface antigen (HBsAg), and hepatitis $\mathrm{C}$ virus were negative.

\section{Radiographic Features}

Magnetic resonance image (MRI) studies revealed a heterogeneous mass with isointense on T1-weighted imaging (Fig. 1a), isointense to hyperintense on T2weighted imaging (Fig. 1b), and hyperintense on fat attenuated imaging (Fig. 1c). The mass was heterogeneous contrast-enhanced and centered in the posterior right orbit, with $57 \times 94 \times 91 \mathrm{~mm}$ in size (Fig. 1d). The lesion was extending laterally into the right medial orbit and the right maxillary sinus with blockage of the osteomeatal complex (Fig. 1e), medially into the right nasal cavity with slight deviation of the nasal septum to the left side, and posteriorly into the nasopharynx (Fig. 1f). Superiorly, the lesion was seen to erode the cribriform plate and extend into the anterior cranial fossa, as well as into the right frontal lobe. There was evidence of peritumoral cysts at the tumor-brain interface with perilesional edema. The lesion involved right ethmoidal, frontal and bilateral sphenoidal sinuses also. Additionally, there was mucosal thickening in the left frontal sinus. No pathologically enlarged retropharyngeal or cervical lymph nodes were noted.

The computed tomography (CT) scans demonstrated a heterogeneously enhancing lobular soft tissue mass that filled the right nasal cavity and orbit (Fig. 1g). Contrary to the clinical impression, the mass did extend posteriorly into the right nasopharynx, causing bowing and thinning of the right maxillary sinus wall. There was erosion of the cribriform plate and crista galli with further invasion into the right frontal sinus and the right medial orbital wall (Fig. 1h). No calcifications were identified in the mass. The left frontal and maxillary sinuses revealed mucosal thickening. There were numerous small unremarkable cervical lymph nodes with no pathologic enlargement.

\section{Histological Characteristics and Immunohistochemical Findings}

Frontal osteoplastic craniotomy and excision of the intracranial part of the tumor from above and transnasal endoscopic removal of the mass in the nasal cavities, paranasal sinuses and right medial orbit from below was done. On histopathological examination, evaluation of the hematoxylin and eosin stained biopsy specimen revealed interconnecting tumor lobules embedded within a richly vascularized fibromyxoid stroma (Fig. 1i). The tumor was composed of lobules, sheets, and nest of primitive cells which were displaying high nuclear:cytoplasmic (N:C) ratio, pleomorphism, round hyperchromatic nuclei with inconspicuous nucleoli, and scanty cytoplasm. On immunohistochemistry, the tumor cells were immunoreactive for neuron-specific enolase (NSE), synaptophysin, chromogranin, CD56, Bcl-6, CD99, and peripherally for 


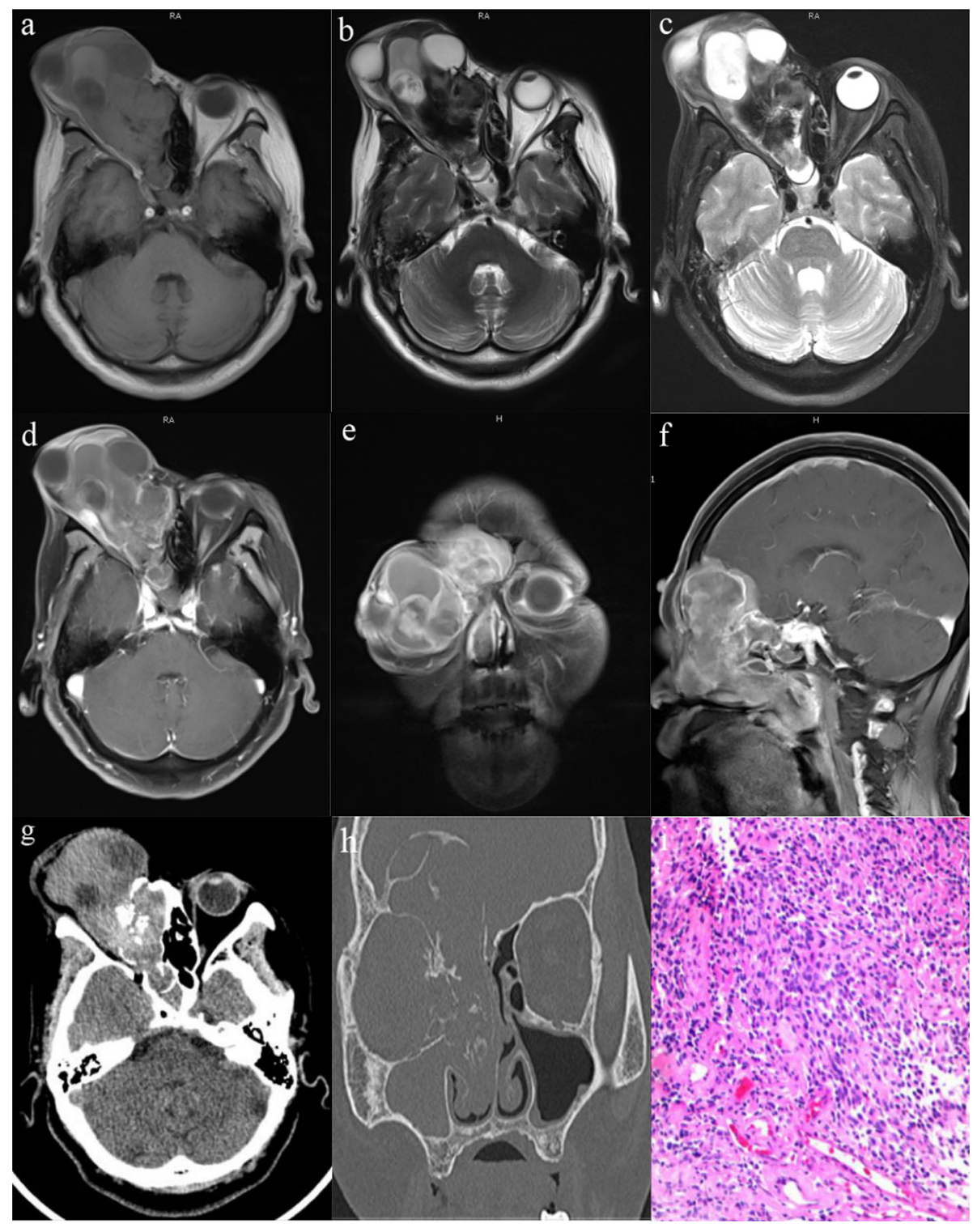

Fig. 1 Pre-operative contrast-enhanced MRI and CT scan of the brain and paranasal sinuses and the histopathological examination with hematoxylin and eosin stained. a-c Non-contrast MRI revealed heterogeneous isointense signal of the mass on T1-weighted sequences and hypointense to hyperintense signal on T2-weighted sequences and fat attenuated sequences, with unclear boundaries and large invasion range. $\mathbf{d}-\mathbf{f}$ Contrast-enhanced MRI showed dumbbell shaped mass with intense heterogeneous post contrast enhancement in the right superior nasal cavity with extensions into the right orbit, anterior cranial fossa and paranasal sinuse. Peritumoral cysts are also noted at the tumor brain interface. $\mathbf{g}, \mathbf{h}$ CT scan of the brain and paranasal sinuses revealed that there was erosion of the right paranasal sinuses wall, the cribriform plate, and the right medial orbital wall with significant bony destruction and radial periosteal reaction. The 'waist' of the dumbbell shaped mass is at the cribriform plate. i Hematoxylin and eosin stained slide $(\times 200)$ - showing olfactory neuroblastoma which is having a high nuclear: cytoplasmic ratio, round hyperchromatic nuclei with inconspicuous nucleoli, scanty cytoplasm, and a richly intercellular vascularized fibromyxoid stroma

\section{Discussion}

Olfactory neuroblastoma (ONB) is a rare malignant tumor of neural crest origin, arising from the olfactory epithelium of the nasal vault [3]. It is a relatively uncommon
S100, but nonreactive for cytokeratin (CK), smooth muscle actin (SMA), and epithelial membrane antigen (EMA). The cytomorphologic and immunophenotypic features were characteristic of a WHO grade IV olfactory neuroblastoma. 
neoplasm, and its clinicopathological presentation remains unclear. Berger and Luc first described this uncommon neoplasm in 1924. Since then, approximately 1200 cases of ONB have been identified [2]. However, ONB centered in the posterior right orbit with prominent orbital protrusion reported in this case is even rare. This disease has a higher degree of malignancy, and the younger the age, the worse the prognosis. Given the pathway of the olfactory nerve, ONB can possiblely invade adjacent structures, such as the ethmoidal sinus, anterior skull base, orbit, and even across the midline to the contralateral nasal cavity [1]. If metastatic, ONB may involve local lymph nodes, with distant metastasis to lungs, liver, and bone.

The most common presenting symptoms are unilateral nasal obstruction (70\%), and epistaxis (50\%). Other symptoms include anosmia, headache, pain, excessive lacrimation, and rhinorrhea. In particular, patients with periorbital extension may present with proptosis, periorbital edema and decrease visual acuity, just like the rare case we reported. Uncommonly, ONB may be associated with syndrome of inappropriate antidiuretic hormone secretion (SIADH) with dilutional hyponatremia or ectopic adrenocorticotropic hormone (ACTH) production leading to Cushing syndrome [4].

ONB may histologically mimic a number of types of tumor within the sinonasal tract, making it much difficult to diagnosis [5]. Multi-modality imaging is essential to correctly assess the extent of the disease in the management of this infrequent tumor [6]. CT and MRI with and without contrast is the first line for evaluation of ONB. The imaging pathway in this case was typical, with CT and MRI complementing each other in maximizing tumor delineation [7]. The CT scan is a helpful initial study to identify the lesion but more importantly, it has superior definition in reviewing bony involvement of the cribriform plate, orbit, and air sinuses [3]. Typically, on CT, a homogenous mass with necrotic nonenhancing areas is observed. As to MRI, ONB usually appears hypointense on T1-weighted sequences and intermediate to hyperintense on T2-weighted sequences. Contrast enhancement is avid and homogeneous, except for areas of necrosis or hemorrhage. MRI has superiority in evaluating the extent of soft tissue invasion and establishing tumor boundaries against post obstruction fluid in the paranasal sinuses. Typically, a dumbbell shaped mass extending across the cribriform plate is one of the most characteristic findings of this tumor [8]. The upper portion of the dumbbell-shaped mass is in the anterior cranial fossa whereas the lower portion is in the nasal cavity with the waist at the cribriform plate [9]. Another characteristic imaging feature of ONB is the presence of peritumoral cysts at the tumor brain interface [2]. In this case, we can also see a dumbbell-shaped mass with the waist at the cribriform plate and peritumoral cyst, which is helpful in the accurate diagnosis of ONB.

Histologically, ONB typically have a high nucleus/cytoplasm ratio, with scant, poorly defined cytoplasm. Highgrade tumors characteristically present with nuclear pleomorphism, mitotic figures $(>2$ per high-power microscope field), and necrosis [10]. Homer Wright rosettes are present in up to $30 \%$ of cases and Flexner-Wintersteiner rosettes are seen in up to $5 \%$. The ultrastructural features of ONB are dense membrane-bound neurosecretory granules with neurofilaments and micro-tubules in the cytoplasm and nerve processes. They typically stain positive for NSE, neurofilament protein, synaptophysin, chromograinin, CD56, and LEU-7. S-100 protein staining seen along the periphery of the neoplastic lobules, also seen in our case, is the characteristic manifestation of ONB. Hematolymphoid markers (CD45, B cell, and $\mathrm{T}$ cell), myogenic markers (myoglobin, desmin, myogenin), melanoma markers (HMB 45, melan A, tyrosinase) and Ewing's sarcoma markers (CD99/MIC2) are absent in most ONB cases. Epithelial markers including EMA, SMA and carcinoembryonic antigen (CEA) are also absent in ONB.

The diagnosis of ONB is extremely challenging as several sinonasal neoplasms must be excluded. The differential diagnosis is widely broad including rhabdomyosarcoma, lymphoma, undifferentiated carcinoma, neuroendocrine carcinoma, melanoma and Ewing' s sarcoma [11]. The differential diagnosis of ONB and neuroendocrine carcinoma was considered as ONB is nonreactive with $\mathrm{CK}$ and TTF-1. ONB can be separated from Ewing' s sarcoma by the presence of an S100 positive sustentacular network in most cases as well as diffuse positivity for neuroendocrine markers and negativity for CD99 [12]. But untypically in this case, the tumor cells were positive for CD99. In addition, the maximum standardized uptake value in the initial PET/CT may be an adjunct to the differential diagnosis of ONB and sinonasal undifferentiated carcinoma [13]. Furthermore, magnetic resonance diffusion kurtosis imaging (DKI) and dynamic contrast enhanced MRI (DCE-MRI) are helpful in distinguishing ONB from nasal squamous cell carcinoma for significantly higher $\mathrm{K}$ values and lower $\mathrm{V}_{\mathrm{e}}$ values in ONB [1]. In particular, ONB can be differentiated from intracranial immature teratoma for the absence of some increased substances including AFP, b-HCG, and PLAP in serum and cerebrospinal fluid [14]. The diagnosis of ONB can be established after a careful evaluation of radiological study, histopathological examination, immunohistochemistry, and cytogenetic analysis [11].

Kadish et al. and Dulguerov et al. proposed ONB classifications based on primary tumor extension and clinicoradiographic data, respectively. Kadish et al. were the first to propose a staging classification for ONB [8]. Morita 
Table 1 Modified Kadish staging classification and Hyams histologic grading system for olfactory neuroblastoma

\begin{tabular}{|c|c|c|c|c|}
\hline \multicolumn{2}{|l|}{ Staging } & \multicolumn{3}{|l|}{ Description } \\
\hline \multicolumn{5}{|c|}{ Modified Kadish classification } \\
\hline A & & \multicolumn{3}{|c|}{ Tumor confined to the nasal cavity } \\
\hline B & & \multicolumn{3}{|c|}{ Tumor extension to the paranasal sinuses } \\
\hline $\mathrm{C}$ & & \multicolumn{3}{|c|}{$\begin{array}{l}\text { Tumor beyond the nasal cavity and paranasal sinuses, including involvement } \\
\text { of the cribriform plate, base of the skull, intracranial cavity, and/or orbit }\end{array}$} \\
\hline $\mathrm{D}$ & & \multicolumn{3}{|c|}{ Tumor with metastases to cervical lymph nodes and/or distant sites } \\
\hline Grade & I & II & III & IV \\
\hline \multicolumn{5}{|c|}{ Hyams histologic grading system } \\
\hline Cytoarchitecture & Lobular & Lobular & \pm & \pm \\
\hline Mitotic rate & 0 & Low & Moderate & High \\
\hline Nuclear Pleomorphism & Absent & Slight & Moderate & Marked \\
\hline Rosettes & \pm & \pm & True Rosettes & None \\
\hline Necrosis & Absent & Absent & Mild & Extensive \\
\hline
\end{tabular}

\pm , may be present or absent

Table 2 The Dulguerov staging system for olfactory neuroblastoma Staging Description

Primary tumor

T1 Tumor involving the nasal cavity and/or paranasal sinuses (excluding sphenoid), sparing the most superior ethmoid

T2 Tumor involving the nasal cavity and/or paranasal sinuses (including the sphenoid) with extension to or erosion of the cribriform plate

T3 Tumor extending into the orbit or protruding into the anterior cranial fossa, without dural involvement

T4 Tumor involving the brain

Lymph nodes

No No cervical lymph node metastasis

N1 Any form of cervical lymph node metastasis

Distant metastasis

M0 No metastases

M1 Distant metastases

et al. modified the Kadish staging to include 4 groups. The modified Kadish staging classification is the most commonly used approach to classify the anatomic extent of the tumor (Table 1). According to modified Kadish staging, our case belongs to group C. The Dulguerov system (Table 2), which uses the TNM classification and includes the imaging data, is preferred by some oncologists and surgeons for recognizing the early involvement of the cribriform plate in the $\mathrm{T} 2$ stage and identifying the true brain involvement. ONB is divided into 4 grades by Hyams based on the following microscopic features: cellular architecture and pleomorphism; mitotic activity; and presence of necrosis, calcification, gland proliferation, and neurofibrillary matrix or rosettes [15]. Histopathology is considered a potentially important prognostication, Hyams grades III and IV are associated with a poor prognosis.

The management of ONB usually requires craniofacial surgical approach, trephination procedure, which is technically challenging and achieving good results are difficult. Recently, authors have come to an agreement that a multimodal approach is necessary to treat patients with ONB. Treatment modalities for ONB are enbloc resection, extra cranial resection or surgery combined with radiotherapy and/or chemotherapy. But unfortunately, the patient did not take adjuvant radiotherapy and chemoradiation and postoperative examination because of poor economic condition.

\section{Conclusions}

We report a huge ONB uncommonly centered in the posterior right orbit with prominent orbital protrusion. From an analysis of the findings in the olfactory neuroblastomas, the diagnosis of ONB should be considered when radiographic imagings reveal a dumbbell-shaped mass in the nasal cavity with peritumoral cyst, and multi-modality imaging are helpful for evaluating the appearance and the extent of ONB, as well as grading this tumor, which may aid therapeutic decisions and improve survival.

Acknowledgements We appreciate those kind persons for their assistance in data collection, language, and writing. 
Authors' Contributions Design, C. L., W. J. X., Y. Z. G., and G. Y.K.; Data acquisition, C. L., W. J. X.; Funding acquisition, Y. Z. G. and G.Y. K.; Writing-original draft, C. L.; Review \& editing, C. L., W. J. X., and G. Y. K. All authors have read and agreed to the published version of the manuscript.

Funding This study was supported by the National Natural Science Foundation of China (81471721, 81471722 and 81641169); the Program for New Century Excellent Talents in University (No. NCET13-0386); the Program for Young Scholars and Innovative Research Team in Sichuan Province (No. 2017TD0005) of China.

Availability of Data and Materials The data that support the findings of this study are available from the corresponding author upon reasonable request.

\section{Compliance with Ethical Standards}

Conflict of interest The authors declare that they have no competing interests.

Ethics Approval and Consent to Participate We declared that the study protocols were approved by the local ethics committee, and written informed consent was obtained from all participants.

Consent for Publication The patient provided informed consent to publish this all presentations of case reports.

Open Access This article is licensed under a Creative Commons Attribution 4.0 International License, which permits use, sharing, adaptation, distribution and reproduction in any medium or format, as long as you give appropriate credit to the original author(s) and the source, provide a link to the Creative Commons licence, and indicate if changes were made. The images or other third party material in this article are included in the article's Creative Commons licence, unless indicated otherwise in a credit line to the material. If material is not included in the article's Creative Commons licence and your intended use is not permitted by statutory regulation or exceeds the permitted use, you will need to obtain permission directly from the copyright holder. To view a copy of this licence, visit http://creativecommons.org/licenses/by/4.0/.

\section{References}

1. Xiao Z, Tang Z, Qiang J, Qian W, Zhong Y, Wang R, Wang J, Wu L, Tang W (2018) Differentiation of olfactory neuroblastomas from nasal squamous cell carcinomas using MR diffusion kurtosis imaging and dynamic contrast-enhanced MRI. J Magn Reson Imaging 47:354-361

2. Gandhoke CS, Dewan A, Gupta D, Syal SK, Jagetia A, Saran RK, Meher R, Srivastava AK, Singh D (2017) A rare case report of mixed olfactory neuroblastoma: carcinoma with review of literature. Surg Neurol Int 8:83

3. Romano N, Federici M, Castaldi A (2019) Imaging of cranial nerves: a pictorial overview. Insights Imaging 10:33

4. Kadoya M, Kurajoh M, Miyoshi A, Shoji T, Terada T, Nakamoto Y, Tsukamoto Y, Moriwaki Y, Hirota S, Koyama H (2018) Ectopic adrenocorticotropic hormone syndrome associated with olfactory neuroblastoma: acquirement of adrenocorticotropic hormone expression during disease course as shown by serial immunohistochemistry examinations. J Int Med Res 46:4760-4768

5. Uslu GH, Canyilmaz E, Zengin AY, Mungan S, Yoney A, Bahadir O, Gocmez H (2015) Olfactory neuroblastoma: a case report. Oncol Lett 10:3651-3654

6. Dublin AB, Bobinski M (2016) Imaging characteristics of olfactory neuroblastoma (esthesioneuroblastoma). J Neurol Surg B Skull Base 77:1-5

7. Konig M, Osnes T, Jebsen P, Evensen JF, Meling TR (2018) Olfactory neuroblastoma: a single-center experience. Neurosurg Rev 41:323-331

8. Fiani B, Quadri SA, Cathel A, Farooqui M, Ramachandran A, Siddiqi I, Ghanchi H, Zafar A, Berman BW, Siddiqi J (2019) Esthesioneuroblastoma: a comprehensive review of diagnosis, management, and current treatment options. World Neurosurg 126:194-211

9. Olmo HR, Stokes SM, Foss RD (2016) Olfactory neuroblastoma: a case report. Head Neck Pathol 10:256-260

10. Sheehan J, Payne R (2016) Esthesioneuroblastomas. In: Winn HR (ed) Youmans and Winn neurological surgery e-book. Elsevier Health Sciences, New York, pp 1284-1292

11. Hussain OA, Aldandan A, Alkhatib A, Alazzeh G, Almomen A (2019) Ewing's sarcoma of the sinonasal tract with orbital extension: a rare case report and literature review. J Surg Case Rep 2019:y362

12. Suzuki T, Yasumatsu R, Nakashima T, Arita S, Yamamoto H, Nakagawa T (2017) Primary Ewing's sarcoma of the sinonasal tract: a case report. Case Rep Oncol 10:91-97

13. Elkhatib AH, Soldatova L, Carrau RL, Hachem RA, Ditzel L, Campbell R, Prevedello DM, Prevedello L, Filho LF, Campbell RG (2017) Role of (18) F-FDG PET/CT differentiating olfactory neuroblastoma from sinonasal undifferentiated carcinoma. Laryngoscope 127:321-324

14. Jiang Y, Mao X, Zhao Y, Fan C (2018) Intracranial immature teratoma invading the nasal cavity mimicking olfactory neuroblastoma. Medicine 97:e11527

15. Goshtasbi K, Abiri A, Abouzari M, Sahyouni R, Wang BY, Tajudeen BA, Hsu F, Cadena G, Kuan EC (2019) Hyams grading as a predictor of metastasis and overall survival in esthesioneuroblastoma: a meta-analysis. Int Forum Allergy Rhinol 9:1054-1062

Publisher's Note Springer Nature remains neutral with regard to jurisdictional claims in published maps and institutional affiliations. 